\title{
Diagnostic value of TREM-1 and TREM-2 expression in bronchoalveolar lavage fluid in sarcoidosis and other lung diseases
}

\author{
Bucova $\mathrm{M}^{1}$, Suchankova $\mathrm{M}^{1}$, Tibenska $\mathrm{E}^{2}$, Majer $\mathrm{I}^{3}$, Novosadova $\mathrm{H}^{3}$, Urban $\mathrm{S}^{3}$ \\ Institute of Immunology, Faculty of Medicine Comenius University Bratislava, Slovakia. \\ maria.bucova@gmail.com
}

\begin{abstract}
BACKGROUND: Triggering receptors expressed on myelocytes (TREM) belong to new molecules with a great role in innate immune system and inflammation. While TREM-1 is known for its pro-inflammatory activity, the TREM-2 has anti-inflammatory activity and has a great impact on granuloma formation, typical sign of sarcoidosis and other granulomatous diseases.

METHODS: In our study, we compared the TREM-1 and TREM-2 receptor expressions on the myeloid cell surfaces in bronchoalveolar lavage fluid in patients with pulmonary sarcoidosis (PS), other interstitial lung diseases (ILD), asthma bronchiale (AB), pneumonia, lung cancers, and Quantiferon TB positive patients.

RESULTS: We found increased number of allTREMvariables (total number, percentage, and mean fluorescence intensity /MFI/) of TREM-1 and TREM-2 positive cells in PS and AB patients compared to the control group of patients with other ILD. In patients with pneumonia, only expression of TREM-1 receptor was increased. In ILD, AB and group of pneumonia patients, the increase of TREM-1 and TREM-2 expression was associated with an increased number of eosinophils. CONCLUSION: TREM-1 and TREM-2 tests are good diagnostic tests for sarcoidosis. Their sensitivity and specificity are comparable with the currently common using test, that of CD4/CD8 ratio. The combination of both tests (CD4/CD8 ratio test together with TREM-1 and TREM-2 tests) resulted in an increased sensitivity and specificity (Tab. 7, Fig. 1, Ref. 28). Text in PDF www.elis.sk.

KEY WORDS: asthma bronchiale, bronchoalveolar lavage fluid, lung diseases, sarcoidosis, TREM-1, TREM-2.
\end{abstract}

\section{Research article}

Research on innate immunity and its regulation has recently focused on pathogen recognition receptors (PRRs). Recognizing and binding pathogen associated molecular patterns (PAMPs) and alarmins from damaged, destroyed or activated cells inside of the body (DAMPS - damage associated molecular patterns), these PRRs induce through intracellular signalling an infectious or sterile inflammatory response.

TREM-1 (triggering receptor expressed on myelocytes) and TREM-2 receptors belong to one of the newer PPRs. They were first described in 2000 on the surface of myeloid cells. Both

${ }^{1}$ Institute of Immunology, Faculty of Medicine Comenius University Bratislava, Slovakia, ${ }^{2}$ Medirex, Ltd., Bratislava, Slovakia, and ${ }^{3}$ Department of Pneumology and Phthisiology, Faculty of Medicine Comenius University, Bratislava, Slovakia

Address for correspondence: M. Bucova, MD, PhD, Institute of Immunology, Faculty of Medicine Comenius University, Spitalska 24, SK-813 72 Bratislava, Slovakia.

Acknowledgement: This contribution/publication is the result of the project implementation: Comenius University in Bratislava Science Park supported by the Research and Development Operational Programme funded by the ERDF. Grant number: ITMS 26240220086.

The study was supported also by grant Vega 1/0833/13.
TREM-1 and TREM-2 belong to the members of the TREM transmembrane glycoproteins belonging to the single immunoglobulin variable ( $\mathrm{IgV})$ domain receptor family. As the cytoplasmic tails of both TREM-1 and TREM-2 lacks signalling motifs, the signalling cascade via ITAM motif of DAP12 is required $(1,2,3)$. While TREM-1/DAP12 signalization has proinflammatory activity, strikingly, the TREM-2/DAP-12 pathway may provide both inhibitory and activating signals depending on their microenvironment $(2,3)$. Moreover, the biological functions of TREM-2/DAP12 pathway may depend on the influence of additional receptors (TLRs), and on the presence of TREM-2 ligands, different cytokines (IL-4, IL-13, IFN- $\gamma$ ), $\beta$-integrins, which may finally induce activating or inhibitory signals through TREM-2/DAP-12 pathway $(2,4,5)$.

There are two forms of these TREM molecules. Besides receptor function, their soluble forms play a great role in the inflammatory response regulation. An increased TREM-1 expression on the surface of myeloid cells occurs in the presence of extracellular bacteria, fungi and their products $(6,7,8)$. Increased TREM-1 expression and increased levels of soluble TREM-1 accompany both infectious and non-infectious inflammatory processes $(9,10)$. TREM-2 is involved in cell fusion and granuloma formation, the typical sign of sarcoidosis (11).

TREM-2 is expressed on osteoclasts, microglial cells, dendritic cells (DCs), tissue infiltrating macrophages from the circulation 


\section{7-713}

(not resident macrophages) and macrophages activated by IL-4 and IL-13 (4). Engagement of TREM-2/DAP-12 under inflammatory conditions have been suggested to promote differentiation of macrophages, DCs and microglial cells. Human TREM-2 regulates the activation of DCs, macrophages, osteoclasts and microglial cells and plays a critical role in fine-tuning inflammatory response (4, $5,12,13)$. TREM-2 is a key negative regulator of autoimmunity, inhibits the production of TNF and IL-6 by macrophages and is responsible for DAP12 mediated inhibition of inflammatory response induced by TLR ligands. TREM-2/DAP12 signalisation triggers the macrophage differentiation and their programming into fusogenic state (14). TREM-2/DAP12 also plays a positive role in phagocytosis, binding both exogenous and endogenous ligands. TREM-2 expression on both microglia and macrophages is associated with specific activated cell phenotype, which performs important protective functions, such as tissue repair, control of local inflammation or phagocytosis of dying cells. TREM-2 can also bind several types of fungi and bacteria, and in association with DAP12 can promote their phagocytosis $(15,16,17,18)$.

In our previous study, we showed an increased expression of both TREM-1 and TREM-2 receptors on the surface of myeloid cells in bronchoalveolar lavage fluid (BALF) in pulmonary sarcoidosis and increased levels of soluble sTREM-1 in BALF of PS patients $(19,20)$. TREM-1 and TREM-2 receptor expressions appear to be a good diagnostic marker with a relatively high sensitivity and specificity (19).

In our present study, we compared TREM-1 and TREM-2 receptor expressions on the myeloid cell surfaces in bronchoalveolar lavage fluid in patients suffering from pulmonary sarcoidosis, other interstitial lung diseases, asthma bronchiale, pneumonia, lung cancers and Quantiferon TB positive patients.

\section{Subjects and methods}

53 patients with pulmonary sarcoidosis (PS), 62 with other interstitial lung diseases (ILD), 8 with allergic asthma bronchiale, 9 with pneumonia, two with lung cancers, and 5 Quantiferon positive patients were prospectively enrolled in the study. All patients were diagnosed at Department of Pneumology and Phthisiology, Comenius University, University Hospital in Bratislava. The study was approved by the Local Ethical Committee of Faculty of Medicine Comenius University in Bratislava and a written informed consent was obtained from all patients.

The diagnosis of PS was established in compliance with criteria recommended by the American Thoracic Society (ATS), the European Respiratory Society (ERS), and the World Association of Sarcoidosis and Other Granulomatous Disorders (WASOG): typical clinico-radiological presentation, the histological evidence of non-caseating granuloma, and/or an increased CD4/CD8 ratio $(>3.5)$ in BALF (21). According to chest radiographic staging, $20 \%$ of PS patients had stage I of the disease, $66 \%$ of patients had stage II, $6 \%$ stage III, and $8 \%$ of patients had stage IV of the disease. None of them received corticosteroid treatment before BAL procedure. Other possible diseases causing granuloma formation were excluded. All PS patients had no comorbidities, negative BALF culture (for aerobic, anaerobic bacteria and yeasts, Mycobacterium tuberculosis culture), negative serologic results (for chlamydias, mycoplasmas and aspergilli).

The group of other ILD consisted of patients with idiopathic pulmonary fibrosis $(47 \%)$, connective tissue related interstitial lung disease (27\%), drug-induced interstitial lung diseases (6 $\%)$, nonspecific interstitial pneumonia (6\%), radiation-induced interstitial lung disease $(\mathrm{n}=5 \%)$, hypersensitivity pneumonitis $(6 \%)$, and histiocytosis $(3 \%)$. Inclusion criteria for this group were radiographic features of fibrosis on HRCT (high resolution computer tomography) scan - GGO (ground-glass opacity) and/ or honeycombing, reticulonodular infiltrates (all patients) and a decreased lung diffusion capacity (all patients).

Asthma bronchiale patients underwent spirometric examinations, bronchoprovocation test, allergen skin tests, and the levels of total IgE were detected, too. Spirometry was measured by pneumotachograph Masterscope Masterscreen (Jaeger). The examination consisted of minimally three valid forced expiratory maneuvers after a maximum inspiration. Expirium lasted at least 8-10 seconds. The largest forced vital capacity (VC) was taken into account. Reference values were according to the publication Kristufek et al (22). The presence of airway obstruction was defined according to the criteria of the European Respiratory Society (23), when the ratio of forced expiratory volume in one second (FEV1) to vital capacity (VC), (FEV1/VC), was less than $88 \%$ of the reference value in men and less than $89 \%$ of the reference value in women. Degrees of obstructive ventilatory disorders were defined according to the decrease of FEV1 (forced expiratory volume in one second) as following: mild degree (mild obstructive ventilatory disorder): $60 \%$ of the reference value-lower limit of normal, moderate degree (moderate obstructive ventilatory disorder): 59 $45 \%$ of the reference value, and severe degree (severe obstructive ventilatory disorder): less than $45 \%$ of the reference value. Bronchoprovocation tests were performed with metacholine according to American Thoracic Society (24). The bronchial hyperreactivity (hyperresponsiveness) was considered when FEV1 fell by $20 \%$ after metacholine dose of $8 \mathrm{mg} / \mathrm{mL}(\mathrm{PC} 20<8 \mathrm{mg} / \mathrm{mL})$. The total plasma IgE level in AB patients was determined by an electrochemiluminescence method (Modular Analytics E170, Roche, USA).

Patients with pneumonia and Quantiferon TB positive patients were selected on the basis of results from anamnesis, laboratory (CRP, leucocytosis, microbial culture, serologic examination, Quantiferon TB gold or T-SPOT TB IGRA tests and CT scans. Patients with lung cancer were diagnosed by $\mathrm{CT}$ scans, histologic results, and blood testing.

BAL procedure during bronchoscopy was carried out at the Department of Pneumology and Phthisiology, Faculty of Medicine Comenius University in Bratislava. BALF harvesting was performed by instillation of $100 \mathrm{ml}$ of sterile normal saline (in two successive $50 \mathrm{ml}$ aliquots) into the right middle lobe and aspirated by gentle suction using a flexible fiberoptic bronchoscope.

BALF was first filtered through a double layer of sterile gauze, centrifuged at $300 \mathrm{~g}$ for $15 \mathrm{~min}$ at $4{ }^{\circ} \mathrm{C}$, supernatants were collected, finally, the pelleted cells were gently resuspended in PBS (phosphate buffer saline) and used for flow cytometric staining. 
TREM-1 and TREM-2 expressions were examined by flow cytometry on cells in freshly isolated BALF $(10-20 \mathrm{ml})$ samples. For the flow cytometric analysis of BALF cells, specimens were double stained with a phycoerythrin (PE)-conjugated mouse anti-human TREM-1 (clone 193015) and rat allophycocyanin (APC)-conjugated anti-human TREM-2 (clone 237920) monoclonal antibodies (R\&D Systems, Minneapolis, MN, USA). An anti-human/mouse IgG1 K isotype control-phycoerythrin conjugated (eBioscience, Inc.) and anti-human/rat IgG2b K isotype control-allophycocyanin conjugated (eBioscience, Inc.) monoclonal immunoglobulins were used. Moreover, staining with fluorescein isothiocyanate (FITC)-conjugated mouse anti-human CD14 (Beckman Coulter Company, Immunotech, Marseille, France) was performed to detect monocyte/macrophages and granulocytes, respectively. Cells were analyzed on a NAVIOS (Beckman Coulter, Inc, USA) cytometer using CXP software (Beckman Coulter, Inc, USA). TREM1 and TREM-2 expressions were measured as the total number and the percentage of TREM-1 and TREM-2 positive cells out of all $\mathrm{CD} 14^{+}$cells and mean fluorescence intensity (MFI) of 10,000 cells in BALF subtracted from isotype control. Total number of TREM-1 and TREM-2 positive cells was calculated from absolute count of BALF cells evaluated by flow cytometer (absolute count of cells per millilitre was obtained of filtered, not concentrated, not centrifuged BALF by Flow-Count Fluor spheres (Beckman Coulter Inc.).

Moreover, all patients were assessed for both total and differential blood cell counts (total numbers of leucocytes, red blood cells, platelets, absolute, and relative number of neutrophils, lymphocytes, monocytes, eosinophils, and basophils by an optic flow cytometry (Sysmex XT 1800i (Sysmex Corporation)). All aforementioned laboratory examinations and also the estimation of Quantiferon tuberculosis tests (QuantiFERON TB GOLD and T-SPOT TB IGRA tests) were done in a specialized biochemical laboratory (Medirex, Ltd., Bratislava).

\section{Statistical analysis}

The one-sample Kolmogorov-Smirnov test was used to determine whether the investigated population followed a normal distribution. Non-parametric Kruskal-Wallis test (non-parametric ANOVA) was used to determine the difference and the statisti- cal significance. Pearson or Spearman test was used to determine data correlation. Fisher's exact test was used to determine the differences between the number of patients with expression higher than the determined cut off and patients with expression lower than the determined cut off. We defined the cut off empirically for total number, percentage proportion and mean fluorescence intensity of TREM-1 or TREM-2 in $40 \%$ of maximal value. The values higher than the selected cut off were considered positive and values below the cut off were designated as negative results. We used min-max data normalization formula for variables: total number, percentage of TREM positive cells and MFI (mean fluorescence intensity).

\section{Results}

Our results show an increased number of either TREM-1 or TREM-2, or both of them on the surfaces on myeloid cells in BALF in some of the selected pulmonary diseases. The total number, the percentage and the mean fluorescence intensity (MFI) of TREM-1 and TREM-2 positive cells were investigated.

Increased TREM-1 and TREM-2 receptor expression in pulmonary sarcoidosis. Increased TREM-1 receptor expression in patients with pneumonia

Estimation of TREM-1 and TREM-2 receptor expression on the myeloid cell surfaces of bronchoalveolar lavage fluid revealed an increased of both TREM-1 and TREM-2 receptor expressions in all tested TREM variables (the total number, percentage, and MFI of TREM-1 and TREM-2 positive cells) in PS patients compared to the control group of patients with other ILD. In patients suffering from pneumonia, expression of TREM-1 receptor (the total number of TREM-1 positive cells) was increased only. No further significant differences were found in any other diagnoses (Tab. 1).

In pulmonary sarcoidosis and in asthma bronchiale patients, a simultaneously increased expression of both TREM-1 and TREM-2 in BALF was found

Further statistical tests revealed the percentage of simultaneously both TREM-1 and TREM-2 positive patients related to

Tab. 1. Comparison of total number of TREM-1 and TREM-2 positive cells in bronchoalveolar lavage fluid of control ILD group of patients with PS. asthma bronchiale, pneumonia, Quantiferon TB positive, and lung cancer patients.

\begin{tabular}{|c|c|c|c|c|c|c|}
\hline & $\begin{array}{c}\text { ILD (CG) } \\
n=62\end{array}$ & $\begin{array}{c}\text { PS } \\
n=53\end{array}$ & $\begin{array}{c}\text { Asthma } \\
\mathrm{n}=8\end{array}$ & $\begin{array}{c}\text { Pneumonia } \\
n=9\end{array}$ & $\begin{array}{l}\text { Quantiferon TB positive } \\
\qquad \mathrm{n}=5\end{array}$ & $\begin{array}{l}\text { Lung cancer } \\
\qquad \mathrm{n}=2\end{array}$ \\
\hline TREM-1 & Mean: 50.9 & Mean: 113.1 & Mean: 64.5 & Mean: 118.2 & Mean: 22.6 & Mean: 31.3 \\
\hline \multirow[t]{2}{*}{ Total number } & Med: 19.2 & Med: 38.4 & Med: 44.1 & Med: 97.8 & Med: 12.8 & Med: 31.3 \\
\hline & $\mathrm{R}: 0.8-728.0$ & $\mathrm{R}: 0.5-2083$ & $\mathrm{R}: 9.3-257.9$ & $\mathrm{R}: 22.2-278.2$ & $\mathrm{R}: 2.2-60.3$ & $\mathrm{R}: 4.0-57.5$ \\
\hline $\mathrm{p}$ & & $<0.05$ & ns & $<0.05$ & ns & \\
\hline TREM-2 & Mean: 26.7 & Mean: 60.8 & Mean: 76.5 & Mean: 21.0 & Mean: 39.5 & Mean: 88.6 \\
\hline \multirow[t]{2}{*}{ Total number } & Med: 10.3 & Med: 27.4 & Med: 22.2 & Med: 18.0 & Med: 40.1 & Med: 88.6 \\
\hline & $\mathrm{R}: 0.3-322.3$ & R:1.7-1240 & $\mathrm{R}: 0.4-404.8$ & $\mathrm{R}: 1.5-53.1$ & R:17.7-59.6 & $\mathrm{R}: 27.8-149.5$ \\
\hline
\end{tabular}

Total number - total number of TREM positive cells; ILD - interstitial lung diseases; CG - control group; PS - pulmonary sarcoidosis; ns - not significant; Med - median, $\mathrm{R}$ - range, $\mathrm{P}$ (Kruskal-Wallis non-parametric ANOVA test); $\mathrm{p}$ value compares the differences between control ILD group with other diagnosis (PS, asthma bronchiale, pneumonia, Quantiferon TB positive patients) 
Tab. 2.Comparison of percentage of TREM-1 and TREM-2 positive cells in bronchoalveolar lavage fluid of control ILD group of patients with PS, asthma bronchiale, pneumonia, Quantiferon TB positive, and lung cancer patients.

\begin{tabular}{|c|c|c|c|c|c|c|}
\hline & $\begin{array}{c}\text { ILD (CG) } \\
n=62\end{array}$ & $\begin{array}{c}\mathrm{PS} \\
\mathrm{n}=53\end{array}$ & $\begin{array}{c}\text { Asthma } \\
\mathrm{n}=8\end{array}$ & $\begin{array}{c}\text { Pneumonia } \\
n=9\end{array}$ & $\begin{array}{l}\text { Quantiferon TB positive } \\
\mathrm{n}=5\end{array}$ & $\begin{array}{l}\text { Lung cancer } \\
n=2\end{array}$ \\
\hline Percentage & Mean: 28.3 & Mean: 49.8 & Mean: 48.2 & Mean: 36.1 & Mean: 16.9 & Mean: 7.9 \\
\hline of TREM-1 & Med: 22.1 & Med: 51.1 & Med: 48.9 & Med: 36.4 & Med: 16.0 & Med: 7.9 \\
\hline+ cells & R:0.4-81.6 & $\mathrm{R}: 1.5-91$ & R:1.8-84.6 & R:8.8-55.3 & R:3.3-39.8 & R:5.2-10.6 \\
\hline $\mathrm{p}$ & & $<0.001$ & ns & ns & $\mathrm{ns}$ & \\
\hline Percentage & Mean: 13.8 & Mean: 34.8 & Mean: 40.5 & Mean: 9.7 & Mean: 37.6 & Mean: 31.4 \\
\hline of TREM-2 & Med: 9.9 & Med: 34.5 & Med: 41.7 & Med: 6.8 & Med: 26.5 & Med: 31.4 \\
\hline+ cells & $\mathrm{R}: 0.3-60.8$ & $\mathrm{R}: 2.7-87$ & $\mathrm{R}: 0.5-98.1$ & $\mathrm{R}: 1.1-32.2$ & $\mathrm{R}: 18.2-72$ & $\mathrm{R}: 27.1-35.7$ \\
\hline
\end{tabular}

$\mathrm{p} \quad<0.001 \quad \mathrm{~ns} \quad \mathrm{~ns} \quad \mathrm{~ns}$

ILD - interstitial lung diseases; CG - control group; PS - pulmonary sarcoidosis; ns - not significant; Med - median, R - range, P (Kruskal-Wallis non-parametric ANOVAtest); P value compares the differences between control ILD group with other diagnosis (PS, asthmabronchiale, pneumonia, Quantiferon TB positive patients).

Tab. 3. Comparison of mean fluorescence intensity of TREM-1 and TREM-2 positive cells in bronchoalveolar lavage fluid of control ILD group of patients with PS, asthma bronchiale, pneumonia, Quantiferon TB positive, and lung cancer patients.

\begin{tabular}{|c|c|c|c|c|c|c|}
\hline & $\begin{array}{c}\text { ILD (CG) } \\
n=62\end{array}$ & $\begin{array}{c}\text { PS } \\
\mathrm{n}=53\end{array}$ & $\begin{array}{c}\text { Asthma } \\
\mathrm{n}=8\end{array}$ & $\begin{array}{c}\text { Pneumonia } \\
n=9\end{array}$ & $\begin{array}{l}\text { Quantiferon TB positive } \\
\qquad \mathrm{n}=5\end{array}$ & $\begin{array}{l}\text { Lung cancer } \\
\quad \mathrm{n}=2\end{array}$ \\
\hline TREM-1 & Mean: 4.5 & Mean:: 9.4 & Mean: 8.7 & Mean: 4.7 & Mean: 2.2 & Mean: 2.8 \\
\hline \multirow[t]{2}{*}{ MFI } & Med: 2.5 & Med: 6.3 & Med: 6.8 & Med: 4.9 & Med:2.8 & Med: 2.8 \\
\hline & $\mathrm{R}: 0.5-27.6$ & $\mathrm{R}: 1.3-45.5$ & $\mathrm{R}: 0.8-23.6$ & $\mathrm{R}: 0.7-8.5$ & $\mathrm{R}: 0.9-3.3$ & $\mathrm{R}: 0.9-4.8$ \\
\hline $\mathrm{p}$ & & $<0.001$ & $\mathrm{~ns}$ & $\mathrm{~ns}$ & ns & \\
\hline TREM-2 & Mean: 5.3 & Mean: 11.3 & Mean: 12.1 & Mean: 1.8 & Mean: 5.7 & Mean: 7.1 \\
\hline \multirow[t]{2}{*}{ MFI } & Med: 2.3 & Med: 4.6 & Med: 5.7 & Med: 1.3 & Med: 4.3 & Med: 7.1 \\
\hline & $\mathrm{R}: 0.6-57.0$ & $\mathrm{R}: 0.8-74.3$ & $\mathrm{R}: 0.7-59.2$ & $\mathrm{R}: 0.6-4.7$ & $\mathrm{R}: 2.0-9.4$ & $\mathrm{R}: 1.9-12.3$ \\
\hline
\end{tabular}

MFI - mean fluorescence intensity of TREM positive cells; ILD - interstitial lung diseases; CG - control group; PS - pulmonary sarcoidosis; ns - not significant; Med - median, $\mathrm{R}$ - range, P (Kruskal-Wallis non-parametric ANOVAtest); $\mathrm{p}$ value compares the differences between control ILD group with other diagnosis (PS, asthmabronchiale, pneumonia, Quantiferon TB positive patients).

Tab. 4. Comparison of percentage of TREM-1 and TREM-2 simultaneously positive patients with diagnoses: ILD (control group), PS, asthma bronchiale, pneumonia, Quantiferon TB positive, and lung cancer patients. Proportion of eosinophil positive patients out of TREM-1 and TREM-2 simultaneously positive patients.

\begin{tabular}{|c|c|c|c|c|c|c|}
\hline & $\begin{array}{l}\mathrm{ILD}(\mathrm{CG}) \\
\mathrm{n}=62\end{array}$ & $\begin{array}{c}\mathrm{PS} \\
\mathrm{n}=53\end{array}$ & $\begin{array}{c}\text { Asthma } \\
\mathrm{n}=8\end{array}$ & $\begin{array}{c}\text { Pneumonia } \\
n=9\end{array}$ & $\begin{array}{l}\text { Quantiferon TB positive } \\
n=5\end{array}$ & $\begin{array}{l}\text { Lung cancer } \\
\quad \mathrm{n}=2\end{array}$ \\
\hline $\begin{array}{l}\text { Percentage of TREM-1 + TREM-2 } \\
\text { positive patients }\end{array}$ & $8 \%$ & $70 \%$ & $75 \%$ & $\begin{array}{c}10 \% \\
\text { (1 patient) }\end{array}$ & $\begin{array}{c}20 \% \\
\text { (1 patient) } \\
\end{array}$ & $0 \%$ \\
\hline $\mathrm{p}$ & & $<0.0001$ & $<0.0001$ & ns & ns & ns \\
\hline Eo positive patients (from TREM-1 & $75 \%$ & $8 \%$ & $40 \%$ & $100 \%$ & $0 \%$ & $0 \%$ \\
\hline
\end{tabular}

and TREM-2 positive)

Percentage of both TREM-1 and TREM-2 positive patients; ILD - interstitial lung diseases; CG - control group; PS - pulmonary sarcoidosis; ns - not significant; Med median; $\mathrm{R}$ - range; P (Fisher test); $\mathrm{p}$ value related to CG (ILD) versus other diagnosis (PS, asthma, Pneumonia, Quantiferon TB positive); percentage of eosinophils - percentage of patients with elevated numbers of eosinophils in BALF and/or blood).

the default cut off. Fisher's test disclosed increased expression of both TREM-1 and TREM-2 receptor in pulmonary sarcoidosis and in asthma bronchiale, respectively (Tab. 4 ). $70 \%$ of pulmonary sarcoidosis and $75 \%$ of asthma bronchiale patients were simultaneously TREM-1 and TREM-2 positive. However, only $20 \%$ of TB patients, $10 \%$ of patients suffering from pneumonia, and $8 \%$ of control ILD patients were both TREM-1 and TREM-2 positive. Interestingly, out of these simultaneously TREM-1 and TREM-2 positive patients, most of them had an increased absolute number of eosinophils (75\% in ILD, $100 \%$ in pneumonia) (Tab. 4).

Increase in TREM-1 and TREM-2 expression is associated with elevated number of eosinophils

Interestingly, as shown in the Table 4, increased TREM-1 and TREM-2 expressions were found not only in patients with sarcoid- osis, but also in those with asthma, other ILD, and pneumonia; the most of them (75\% ILD, $40 \%$ asthma, $100 \%$ pneumonia) had elevated eosinophils counts (blood and/or BALF). Further statistical tests revealed a significant positive correlation between increased TREM-1 and TREM-2 expression and the number of eosinophils in "non-sarcoidosis" patients (asthma, ILD, pneumonia, Quantiferon TB positive, lung cancer). No significant correlation was found between increased TREM-1 and TREM-2 expression and the number of eosinophils in sarcoidosis patients (Tab. 5).

TREM-1 and TREM-2 test as a diagnostic tests for sarcoidosis. Sensitivity and specificity of TREM-1 and TREM-2 tests is comparable to the common using test - CD4/CD8 ratio

$\mathrm{CD} 4 / \mathrm{CD} 8$ ratio is a commonly used diagnostic test for diagnosis of PS for a long time. The sensitivity and specificity varies 
Tab. 5. Correlation between TREM-1 and TREM-2 expressions on the myeloid cell surfaces in BALF and number of eosinophils (BALF and/or blood).

\begin{tabular}{lcc}
\hline $\begin{array}{l}\text { Absolute number } \\
\text { of eosinophils }\end{array}$ & $\begin{array}{c}\text { TREM-1 + TREM-2 } \\
\text { (sarcoidosis) }\end{array}$ & $\begin{array}{c}\text { TREM-1 + TREM-2 } \\
\text { (ILD, asthma, pneumonia, Quan- } \\
\text { tiferon TB positive, lung cancer) }\end{array}$ \\
\hline $\mathrm{R}$ & 0.08118 & 0.3355 \\
\hline $95 \% \mathrm{CI}$ & $-0.2099-0.3590$ & $0.1365-0.5084$ \\
\hline $\mathrm{p}$ & 0.5752 & 0.0009 \\
\hline
\end{tabular}

Number of eosinophils is calculated as sum of blood and BALF numbers expressed in normalised data transformation ( $\min -\max$ data normalisation method); TREM-1 + TREM-2 - total expression calculated as sum of total number, percentage and MFI of TREM-1 and TREM- 2 expressed in a scale $0-1$ using min-max normalization method; P: Spearman test ( $\mathrm{x}$ - Eosinophils, y1 - TREM-1, y2 - TREM-2).

Tab. 6. Sensitivity and specificity of CD4/CD8 ratio test of pulmonary sarcoidosis patients.

\begin{tabular}{lcc}
\hline & $\begin{array}{c}\text { CD4/CD8 Cut off }>3.5 \\
\text { (from literature) }\end{array}$ & $\begin{array}{c}\text { CD4/CD8 Cut off }>3.5 \\
\text { (studied patients) }\end{array}$ \\
\hline Sensitivity & $52-59 \%$ & $60 \%$ \\
\hline Specificity & $94-96 \%$ & $96 \%$ \\
\hline
\end{tabular}

Tab. 7. Sensitivity and specificity of TREM-1 and TREM-2 test for patients with pulmonary sarcoidosis.

\begin{tabular}{|c|c|c|c|}
\hline $\begin{array}{l}\text { TREM-1 + TREM-2 test; } \\
>2 \text { positive: } \\
\text { 1. total number } \\
\text { 2. Percentage } \\
\text { 3. MFI }\end{array}$ & Sarcoidosis & ILD & $\begin{array}{c}\text { ILD } \\
\text { Eonegat. }\end{array}$ \\
\hline Positive patients (\%) & 70 & 8 & 2 \\
\hline $\mathrm{p}$ & & $<0.0001$ & $<0.0001$ \\
\hline Sensitivity (\%) & & 70 & 70 \\
\hline Specificity (\%) & & 92 & 98 \\
\hline
\end{tabular}

TREM-1 + TREM-2 tests: both TREM-1 and TREM-2 positive results - if more than two variables are positive (variables: total number, percentage and MFI of TREMpositive cells); ILD - other interstitial lung diseases; Eonegat. - patients without elevated eosinophils; P (Fisher's test); black arrows: turnover of specificity and percentage of positive patients when eosinophil inflammation is excluded.

according various studies: value over 3.5 has the sensitivity from 52 to $59 \%$ and specificity from 94 to $96 \%$ (25). In our study, the calculated sensitivity and specificity for group of PS patients was $60 \%$ for sensitivity and $96 \%$ for specificity (Tab. 6). We calculated the sensitivity and specificity also for simultaneously positive TREM-1 and TREM-2 tests. The value of sensitivity was 70 $\%$ and specificity $92 \%$. Excluding diagnoses accompanying by increased number of eosinophils, the specificity of the TREM-1 and TREM-2 tests increased from $92 \%$ to $98 \%$ (Tab. 7).

Test combinations. Taking into account more various tests lead to an increased sensitivity and specificity

Taking into account more various tests (Fig. 1), led to their increased sensitivity and specificity for pulmonary sarcoidosis diagnosis. Interestingly, the combination of CD4/CD8 ratio test together with that of TREM-1 and TREM-2 minimally in one variable (variables: total number, percentage and MFI of TREM positive cells) resulted in an increased specificity to $100 \%$ with the sensitivity $55 \%$. Further interesting combination was the positivity of minimally two tests from the following: 1) CD4/CD8 test, 2) the total number of TREM-1 and TREM-2 positive cells, 3) percentage of TREM-1 and TREM-2 positive cells, 4) MFI of TREM-1 and TREM-2 positive cells. In this case, we reached the specificity $98 \%$ and the sensitivity $81 \%$.

\section{Discussion}

Our study has brought interesting results. Comparing TREM1 and TREM-2 receptor expressions on myeloid cell surfaces in bronchoalveolar lavage fluid of patients with pulmonary sarcoidosis, other ILD, asthma bronchiale, pneumonia, lung cancers and Quantiferon TB positive patients, we found an increased number of all TREM variables (total number, percentage, and mean fluorescence TREM-1 and intensity /MFI/) of TREM-2 positive cells in PS and AB patients compared with the control group of patients suffering from other ILD. In patients with pneumonia, the expression of TREM-1 receptor was increased only. In ILD, $\mathrm{AB}$ and group of pneumonia patients, the increased expression of TREM-1 and TREM-2 was associated with an elevated number of eosinophils and thus eosinophilic inflammation. TREM-1 and TREM-2 tests seem to be good diagnostic tests for pulmonary sarcoidosis. Their sensitivity and specificity are comparable to the common using test - CD4/CD8 ratio. The combination of CD4/

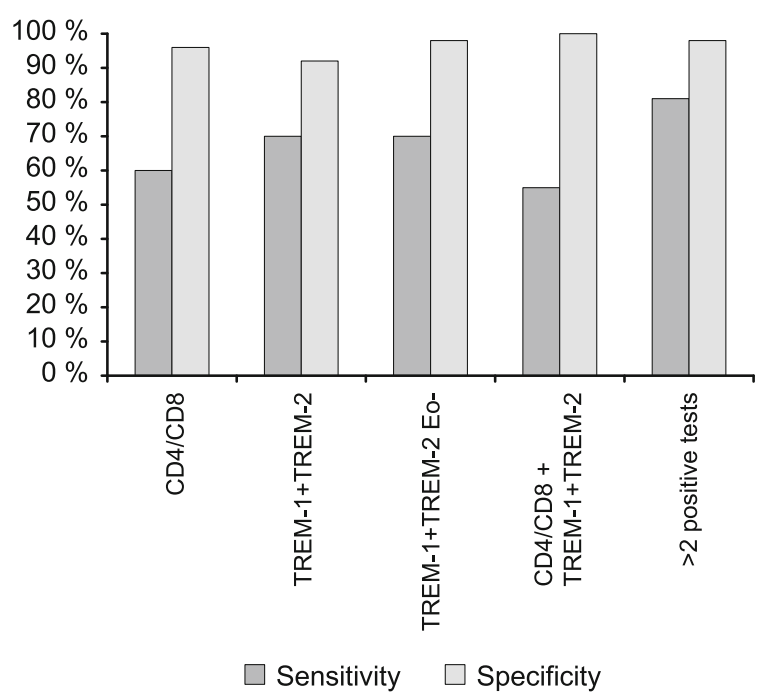

Fig. 1. Comparison of sensitivity and specificity for CD4/CD8 test, TREM-1 + TREM-2 tests and for combination of these tests in pulmonary sarcoidosis compared with other ILD patients. CD4/CD8 - test of CD4/CD8 ratio > 3.5; TREM-1+TREM-2 - test of two or more positive variables (variables: total number, percentage and MFI of TREM-1 and TREM-2 positive cells); TREM-1+TREM-2 Eo- test - two or more positive variables (variables: total number, percentage and MFI of TREM-1 and TREM-2 positive cells) without patients with elevated eosinophils; CD4/CD8 + TREM-1+TREM-2 - combination of tests number of positivity of CD4/CD8 ratio together with TREM-1 and TREM-2 test minimally in one variable (variables: total number, percentage and MFI of TREM positive cells); > 2 positive tests - positivity of minimally two tests from the following: 1. CD4/CD8, 2. total number of TREM-1 and TREM-2 positive cells, 3. percentage of TREM-1 and TREM-2 positive cells, 4.MFI of TREM-1 and TREM-2 positive cells. 
CD8 ratio test together with TREM-1 and TREM-2 test resulted in an increased sensitivity and specificity. Significant association of increased TREM-1 and TREM-2 expression on myeloid cell surfaces in BALF of PS patients has been detected also in our previous study (19). According to these two studies, we might conclude, that increase in both of these receptors are typical for PS diagnosis and can represent a new diagnostic marker for PS. We cannot discuss our obtained results concerning expression of TREM-1 and TREM-2 in BALF in PS patients, as no other studies in PS patients have been published yet.

In our group of PS patients, the BALF level of soluble TREM1 was also investigated. We found four times higher levels of BALF sTREM-1 in PS compared to the control group of other ILD patients $(p=0.001)$. We also recorded the effect of smoking; ILD smokers had higher sTREM-1 levels than non-smokers $(p=0.0019)$. Higher concentrations of sTREM-1 were detected in BALF of patients with lymphadenopathy and with elevated inflammatory markers in BALF. Our results showed, that BALF sTREM-1 could be a good inflammatory marker and could help in diagnosis and PS monitoring (20). Detection of sTREM-1 in BALF indirectly points to myeloid cells activation in the lungs and helps to complete the information about the number of myeloid cells commonly determined in BALF with additional information concerning the intensity of their activation.

In another study, the plasma levels of sTREM-1 in allergic asthma bronchiale patients were tested. Our results revealed more than 3.5 times higher levels of sTREM-1 in AB patients compared with healthy subjects. Higher levels of sTREM-1 were found also in patients with exacerbated $\mathrm{AB}$ compared with nonexacerbated $\mathrm{AB}$ patients, patients with respiratory tract obstruction, than those without obstruction and patients with anti-IgE therapy. Levels of sTREM-1 correlated with the number of leucocytes and the absolute number of neutrophils. Elevated plasma levels of sTREM-1 reflected the severity, state of exacerbation, presence of respiratory tract obstruction in $\mathrm{AB}$ patients and together with an increased number of neutrophils pointed to the role of neutrophils in inflammation accompanying AB (10). Both these studies were the first, which analysed the BALF sTREM-1 levels in patients with PS and plasma levels of sTREM-1 in asthma bronchiale patients.

There are only a few studies analyzing TREM-1 and TREM-2 expressions in BALF. Grover et al. found increased expressions of TREM-1 on monocytes and neutrophils both in BALF and blood in patients with ventilator-associated pneumonia compared to non ventilator-associated pneumonia and non ventilated controls (26). Very interesting are the results of Chan and his colleagues (27). They measured the expression of TREM-1 and the level of sTREM-1 in pleural effusions due to transudate, malignancy and tuberculous pleuritis and found, that the expression of TREM-1 was highest in para-pneumonic effusion and lowest in malignant pleural effusion and tuberculous pleuritis. The highest levels of STREM-1 were measured in effusions of infectious aetiology (parapneumonic effusions and empyema) and lowest in non-infectious effusions - transudates and malignancy (27). There are only a few studies analysing TREM receptors expression in lung cancer. Yuan found an increased expression of TREM-1 in tumour tissues from patients with non-small cell lung cancer (28). No studies investigating TREM-2 receptor in lung cancer have been published yet.

\section{Conclusion}

Our study, that analysed the expression of TREM-1 and TREM-2 molecules on the surfaces of myeloid cells in BALF, showed that TREM-1 and TREM-2 tests seemed to be good diagnostic tests for pulmonary sarcoidosis. Their sensitivity and specificity were comparable with the common using testCD4/ CD8 ratio. Combination of the CD4/CD8 ratio test together with the TREM-1 and TREM-2 tests resulted in an increased sensitivity and specificity.

\section{References}

1. Bouchon A, Dietrich J, Colonna M. Cutting edge: inflammatory responses can be triggered by TREM-1, a novel receptor expressed on neutrophils and monocytes. J Immunol 2000; 164: 4991-4995.

2. Paradowska-Gorycka A, Jurkowska M. Structure, expression pattern and biological activity of molecular complex TREM-2/DAP12. Human Immunol 2013; 74 (6): 730-737.

3. Sharif O, Knapp S. From expression to signalling: Roles of TREM1 and TREM-2 in innate immunity and bacterial infection. Immunobiol 2008; 213: 701-713.

4. Turnbull IR, Gilfillan S, Cella $M$ et al. Cutting edge: TREM-2 attenuates macrophage activation. J Immunol 2006; 177 (6): 3520-3524.

5. Pelham CJ, Agrawal DK. Emerging roles for triggering receptor expressed on myeloid cells receptor family signalling in inflammatory diseases. Expert Rev Clin Immunol 2014; 10 (2): 243-256.

6. Richeldi L, Mariani M, Losi M et al. Triggering receptor expressed on myeloid cells: role in the diagnosis of lung infections. Eur Respir $\mathbf{J}$ 2004; 24 (2): 247-250.

7. Determann RM, Millo JL, Gibo S et al. Serial changes in soluble triggering receptor expressed on myeloid cells in the lung during development of ventilator-associated pneumonia. Intens Care Med 2005; 31 (11): $1495-1500$.

8. Liu CL, Hsieh WY, Wu CL, Kuo HT, Lu YT. Triggering receptor expressed on myeloid cells-1 in pleural effusions: a marker of inflammatory disease. Resp Med 2007; 101 (5): 903-909.

9. Ferat-Osario E, Esquivel-Callejas N, Wong Baeza I et al. The increased expression ofTREM-1 on monocytes is associated with infectious and non-infectious inflammatory processes. J Surg Res 2008; 150: 110-117.

10. Bucova M, Suchankova M, Dzurilla $M$ et al. Inflammatory marker sTREM-1 reflects the clinical stage and respiratory tract obstruction in allergic asthma bronchiale patients and correlates with number of neutrophils. Mediators Inflamm 2012; 2012; 2012: 628754. doi: 10.1155/ 2012/628754.

11. Hamerman JA, Jarjoura JR, Humphrey MB, Nakamura MC, Seaman WE, Lanier LL. Cutting edge: inhibition of TLR and FcR responses in macrophages by triggering receptor expressed on myeloid cells (TREM)2 and DAP12. J Immunol 2006; 177 (4): 2051- 2055.

12. Hiasa M, Abe M, Nakano A et al. GM-CSF and IL-4 induce dendritic cell differentiation and disrupt osteoclastogenesis through M-CSF receptor shedding by up-regulation of TNF-alpha converting enzyme (TACE). Blood 2009; 114 (20): 4517-4526. 
13. Ford JW, McVicar D. TREM and TREM like receptors in inflammation and disease. Curr Opin Immunol 2009; 21: 38-46.

14. Helming L, Tomasello E, Kyriakides TR et al. Essential role of DAP12 signaling in macrophage programming into a fusion-competent state. Sci Signal 2008; 1 (43): ra11. doi: 10.1126/scisignal. 1159665.

15. N'Diaye EN, Branda CS, Branda SS et al. TREM-2 (triggering receptor expressed on myeloid cells 2) is a phagocytic receptor for bacteria. J Cell Biol 2009; 184 (2): 215-223. doi: 10.1083/jcb. 200808080.

16. Gawish R, Martins R, BöhmB et al. Triggering receptor expressed on myeloid cells-2 fine-tunes inflammatory responses in murine Gram-negative sepsis. FASEB J 2014; Dec 4. pii: fj. 14-260067. [Epub ahead of print]

17. Chen $\mathbf{Q}$, Zhang K, Jin $\mathbf{Y}$ et al. Triggering receptor expressed on myeloid cells-2 protects against polymicrobial sepsis by enhancing bacterial clearance. Am J Resp Crit Care 2013; 188 (2): 201-212.

18. Takahashi K, Rochford CD, Neumann H. Clearence of apoptotic neurons without inflammation by microglial triggering receptor expressed on myeloid cells-2. J Exp Med 2005; 201: 247-257.

19. Suchankova M, Bucova M, Tibenska E et al. , Triggering receptor expressed on myeloid cells-1 and 2 in bronchoalveolar lavage fluid in pulmonary sarcoidosis. Respirology 2013; 18 (3): 455-462.

20. Suchankova M, Bucova M, Demian J et al. sTREM-1 in bronchoalveolar lavage fluid in patients with pulmonary sarcoidosis, effect of smoking and inflammation. Bratisl Lek Listy 2013; 114 (12): 702-707.

21. American Thoracic Society/European Respiratory Society and the World Association of Sarcoidosis and Other Granulomatous Disorders (WASOG). Statement on sarcoidosis. Am J Resp Crit Care Med 1999; 160: 736-755.
22. Kristufek P, Brezina M, Ciutti P. Reference values and modelling of lung function development as a transcendent function of age, body height and mass. Clin Resp Physiol 1987; 23 (2): 139-147.

23. Siafakas NM, Vermeire P, Pride NB et al. Optimal assessment and management of chronic obstructive pulmonary disease (COPD). Eur Resp J 1995; 8 (8): 1398-1420.

24. Crapo RO, Casaburi R, Coates AL et al. Guidelines for methacholine and exercise challenge testing - 1999. Am J Resp Crit Care Med 2000; 161 (1): 309-329.

25. Drent M, Mansour K, Linssen C. Bronchoalveolar lavage in sarcoidosis. Semin Resp Crit Care Med 2007; 28 (5): 486-495.

26. Grover V, Pantelidis P, Soni N, Takata M, Shah PL, Wells AU et al. A biomarker panel (Bioscore) incorporating monocytic surface and soluble TREM-1 has high discriminative value for ventilator-associated pneumonia: a prospective observational study. PLoS One 2014; 9 (10): e109686. doi: 10.1371/journal.pone.0109686. eCollection 2014.

27. Chan MC, Chang KM, Chao WC, Lin LY, Kuo BI, Hsu JY et al. Evaluation of a new inflammatory molecule (triggering receptor expressed on myeloid cells-1) in the diagnosis of pleural effusion. Respirol 2007; 12 (3): 333-338.

28. Yuan Z, Mehta HJ, Mohammed K, Nasreen N, Roman R, Brantly $\mathbf{M}$ et al. TREM-1 is induced in tumor associated macrophages by cyclooxygenase pathway in human non-small cell lung cancer. PLoS One 2014; 19 (5): e94241. doi: 10.1371/journal.pone.0094241. eCollection 2014.

Received January 30, 2015. Accepted February 6, 2015. 